\title{
Image Dehazing (Defogging) by using Depth Estimation and Fusion with Guided Filter
}

\author{
Anil Kumar \\ Scope college of Engineering, \\ Bhopal, India
}

\author{
Bharti Chourasia \\ Scope college of Engineering, \\ Bhopal, India
}

\begin{abstract}
The season affects the imaging of the hill station highly and all other reasons moreover time to time. Our universal defoging method that determining the atmospheric light and produces a spread map in the $\mathrm{YCbCr}$ color channels. With this relative depth information we can construct the corresponding atmospheric light to restrain the edge halation. We generate the spread map by estimating the atmospheric light except a continuous region which has no edge information. The method performs a per-pixel manipulation, which is straightforward to implement and then apply the Guided filter to improve the image quality. The experimental results demonstrate that the method yields results comparative to and even better than the more complex state-of-the-art techniques, having the advantage of being appropriate for real-time applications.
\end{abstract}

\section{Keywords}

Image Fusion, Image Defogging, Scattering model, single image defogging

\section{INTRODUCTION}

Haze is an annoying factor when it shows up in the image since it causes poor visibility. This is the major problem of some applications in the field of computer vision, such as surveillance, object recognition, etc. Zhen et al. [2] combined the bilateral filter and the adaptive median filter for clear dark channel on image edges. The physical model of algorithm for foggy images is to estimate transmission. It provides the reliability for outdoor visual systems in foggy climate. Wang et al. [3] proposed a multi values depth fusion (MDF) for recovery of fog using local Markov regularization via single foggy image. The fog priors are fused in the inhomogeneous Laplacian IL-MRF adaptively from multiscale filtering. The depth map estimation is a reiterative process with optimization of two variables in adaptive truncated Laplacian (ATL) potential: a base potential variable to regularize smoothness and a line field variable for adaptive control. In 2014 Kawarabuki et al. [4] presented the snowfall estimation from falling snow grains measurement quality that extracted the difference in present defogged image and image background produced by the median. It recognizes the degree of snowfall automatically even in the low visibility by fog. In 2015 Zhao et al. [5] removed the fog by Image defogging (IDF) method that influences from the fogs in an image to improve its quality. For the single scattering of light dominated pixels the single scattering physics model is used in the hybrid model and for the remaining pixels the multiple scattering physics model (MSPM) is used.

[7] In this paper, we propose a new transmission model using LO norm for image fog removal. In the prior work, the bilateral filter was used to reduce the halo artifacts. However, it is only a local optimization. Hence, we observe non-zero gradients to develop the gradient smoothing method for global control. The proposed model then locates significant edges to highlight the prominent parts of an image. Experimental results show the effectiveness of the proposed model for defogging [7]. The theoretical background can be discussed in terms of the basic image features and the image properties [818]. [19] Image has important applications in many fields such as marine surveillance, environment monitoring and so on. The scattering effects of the atmospheric particles in the air play a main role of resulting in contrast reduction and color fading. For dealing with this challenging but imperative issue, there are numerous researchers have strove for this scientific field and published a plenty of findings about restoring the foggy image. In this paper, we summarized the existing outcomes and reviewed the approaches which were employed for defoging. Based on our best knowledge, we classified the numerous image defoging approaches into two broad categories i.e. image enhancement and physical model restoration. Some classical and prominent defoging approaches were analyzed and elaborated in the term of basic principle, time consuming and typical application field. The validation of performance measurement also was discussed in detail. [20] A wavelet-based Retinex algorithm for unmanned aerial vehicle image defogging is proposed to avoid edge fuzziness, image dinginess, noise pollution and inefficiency, etc. in classical Retinex. In light of different energy distribution of fog, noise and edge information of subjects in the wavelet domain, this algorithm uses Retinex to restrain the influence of the fog, and adopts the wavelet threshold method to restrain noise while preserving the edge information, in order to reduce the influence of fog and noise of image. [21] To overcome the weakness of original dark channel prior algorithm on losing effectiveness in bright regions, an adaptive defogging algorithm was proposed. In this method, the fuzzy logic controller was adopted to automatically estimate the threshold of bright regions and the adjustment factor of transmission, and the misestimated transmission in bright regions was corrected using the adaptive threshold. [22] The fog images of Inland River often have low contrast. This paper introduces a optimized contrast enhancement algorithm. Firstly, divide the fog image by using the hierarchical searching method of quad-tree subdivision. Choose the brightest pixel as the atmospheric light in the final selected block. Then, combine the Mean Squared Error and the loss function. Next, get the optimal transmission value which can balance the contrast and the information loss. Then, fix the transmission with guide filter and adaptive window slide. Finally, restore the defogged image by solving the atmosphere attenuation model. [23] Image defogging has recently received attentions in many applications such as advanced drive assistance systems (ADAS) and intelligent surveillance systems to acquire high-quality images. This paper presents a novel depth-based image defogging method using stereo images. The depth information obtained from a pair of stereo foggy images and then fuzzy C-mean (FCM) clustering is applied to reduce matching errors caused by atmospheric 
absorption and scattering during light propagation. The estimated depth information is used as weighting values in the dark channel prior (DCP)-in the defogging process. [24] Image defogging is a technique used extensively for enhancing visual quality of images in bad weather conditions. Even though defogging algorithms have been well studied, defogging performance is degraded by demosaicking artifacts and sensor noise amplification in distant scenes. It validate the theoretical analysis and observations for both synthesized datasets with ground truth fog-free images and natural scene datasets captured in a raw format. The rest of the paper is organised as follows. The second section provides the methodology on hybrid image defogging algorithm and the Depth fusion based defogging algorithm. The third section shows the result analysis and fourth section covering future scope with in conclusion.

\section{PROPOSED METHODOLOGY}

Image acquisition: Take the input image as we perform all possible operation on it. Figure 1 showing all the procedure related to haze removing algorithm.

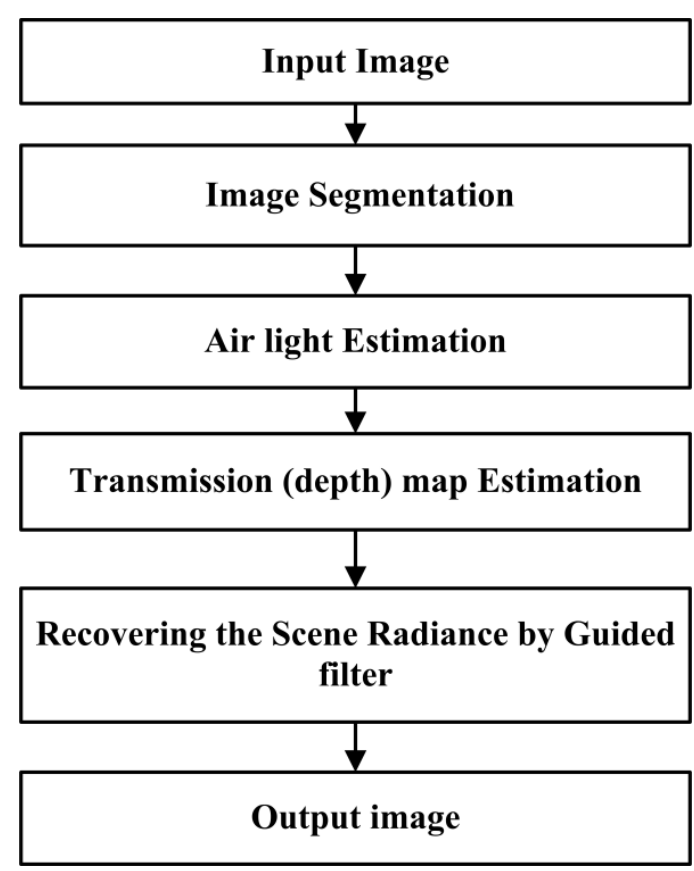

Fig 1: Flowchart of the proposed method

Here we describe the dehazing process in detail. The rough down-sampled transmission and the air-light are estimated firstly, and then the transmission is smoothed and up sampled using a guided filter, and finally the haze-free image is restored.

\subsection{Extract the Transmission}

The core of haze removal for an image is to estimate the airlight and transmission map. Assuming the airlight is already known, to recover the haze free image, the transmission map should be extracted first. He et al. [25] found that the minimum intensity in the non-sky patches on haze free outdoor images should have a very low value, which is called dark channel prior. Formally, for an image $J$, the dark channel value of a pixel $\mathrm{x}$ is defined as:

$$
J^{\text {dark }}(\mathrm{x})=\min _{c \in\{r, g, b\}}\left(\min _{y \in \Omega(x)}\left(J^{c}(y)\right)\right)
$$

where, $J^{c}$ is a color channel of $\mathrm{J} ; \Omega(\mathrm{x})$ is a patch around $\mathrm{x}$. By assuming the transmission in a local patch is constant and taking the min operation to both the patch and three color channels, the haze imaging model in (1) can be transformed as:

$$
\begin{gathered}
\min _{c \in\{r, g, b\}}\left(\min _{y \in \Omega(x)}\left(\frac{I^{c}(y)}{A^{c}}\right)\right) \\
=\tilde{t}(\mathrm{x}) \min _{c \in\{r, g, b\}}\left(\min _{y \in \Omega(x)}\left(\frac{I^{c}(y)}{A^{c}}\right)\right)+(1-\tilde{t}(\mathrm{x}))
\end{gathered}
$$

where, $\tilde{t}(x)$ is the patch transmission. Since $A$ is always positive and the dark channel value of a haze-free image $J$ tends to be zero according to the dark channel prior, we have

$$
\min _{c \in\{r, g, b\}}\left(\min _{y \in \Omega(x)}\left(\frac{I^{c}(y)}{A^{c}}\right)\right) \rightarrow 0
$$

Then the transmission can be exacted simply by:

$$
\tilde{t}(\mathrm{x})=\min _{c \epsilon\{r, g, b\}}\left(\min _{y \in \Omega(x)}\left(\frac{I^{c}(y)}{A^{c}}\right)\right)
$$

Although the dark channel prior is not a good prior for the sky regions, fortunately, both sky regions and non-sky regions can be well handled by (4) since the sky is infinitely distant and its transmission is indeed close to zero. In practice, the atmosphere is not absolutely free of any particle even in clear weather. Therefore, a constant parameter $\omega(0<\omega \leq 1)$ is introduced into (4) to keep a small amount of haze for the distant objects:

$$
\tilde{t}(\mathrm{x})=1-\omega \min _{c \in\{r, g, b\}}\left(\min _{y \in \Omega(x)}\left(\frac{I^{c}(y)}{A^{c}}\right)\right)
$$

The estimated transmission maps using (5) is reasonable. The main problems are some halos and block artifacts. This is because the transmission is not always constant in a patch. Several techniques were proposed to refine the transmission map, such as soft matting and guided joint bilateral filter. These techniques were applied on the transmission maps of the original foggy images and usually several operations should be used to achieve a good result, which could be computational intensive. For image haze removal, the time complexity is a critical problem that needs to be addressed. High time complexity of dehazing may make the algorithm impracticable.

\subsection{Refine the Transmission}

To improve the efficiency, in the present implementation, the transmission map is obtained from a down-sampled minimum channel image. Then, it is refined and up-sampled by using guided filter, which can be explicitly expressed by [28, 29]:

$$
\begin{aligned}
& t_{i}=\sum_{j} W_{i j}\left(J^{g}\right) \tilde{t}_{j} \\
& W_{i j}\left(J^{g}\right)=\frac{1}{|w|^{2}} \sum_{k:(i, j) \in w_{k}}\left(1+\frac{\left(J_{i-\mu_{k}}^{g}\right)\left(J_{j-\mu_{k}}^{g}\right)}{\sigma_{k}^{2}+\varepsilon}\right)
\end{aligned}
$$

Where, $J_{g}$ is the guidance image; $\mu_{k}$ and $\sigma_{k}^{2}$ are the mean and variance of $J_{g}$ in $w_{k} ;|w|$ is the number of pixels in $w_{k}$. $\varepsilon$ is a regularization parameter. The refined operation on a downsampled minimum channel image leads to a low time complexity and helps to reduce halos and block artifacts. Joint upsampling using guided filter is applied to obtain the full transmission map. The guided filter is reported to be a fast and non-approximate linear-time algorithm, which can perform as an edge preserving smoothing operator like the bilateral filter, but does not suffer from the gradient reversal artifacts. Moreover, the guided filter has an $\mathrm{O}(\mathrm{N})$ time (in the number of pixels $\mathrm{N}$ )exact algorithm for both gray-scale and color images. 


\subsection{Recovering the Scene Radiance}

After the transmission map is estimated, the scene radiance can be recovered according $t_{o}(1)$. The term $\mathrm{J}(\mathrm{x}) \mathrm{t}(\mathrm{x})$ can be very close to zero. When the transmission $\mathrm{t}(\mathrm{x})$ is close to zero, which make the recovered scene radiance $\mathrm{J}$ is prone to noise. Therefore, the transmission $\mathrm{t}(\mathrm{x})$ is restricted by a low bound $t_{o}$. The final scene radiance $\mathrm{J}(\mathrm{x})$ is recovered by

$$
J(x)=\frac{I(x)-A}{\max \left(t(x), t_{o}\right)}+A
$$

Where, $\mathrm{A}$ is the airlight. There are many ways available in literatures to estimate the airlight [26, 27]. For simplicity, A takes the value of a pixel with highest dark channel value in this paper.

\section{RESULTS AND DISCUSSION}

The algorithm proposed here will remove haze from an image surface without prior knowledge of the haze location upon

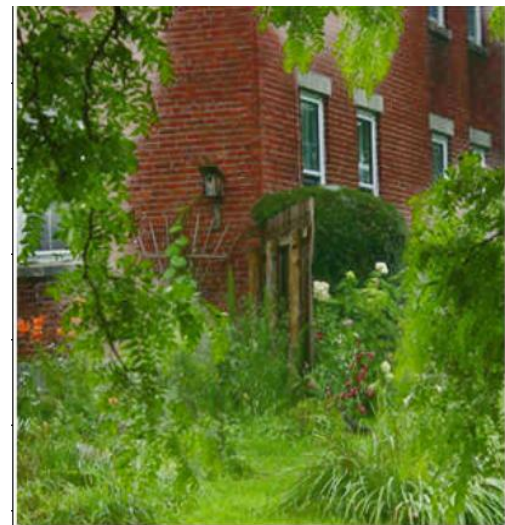

(a)

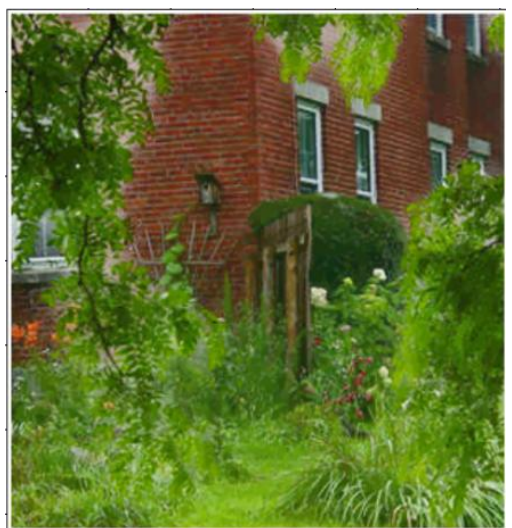

(b) that surface. The proposed method is based on determining the illumination profile of the image surface. This profile is then used to remove the haze.
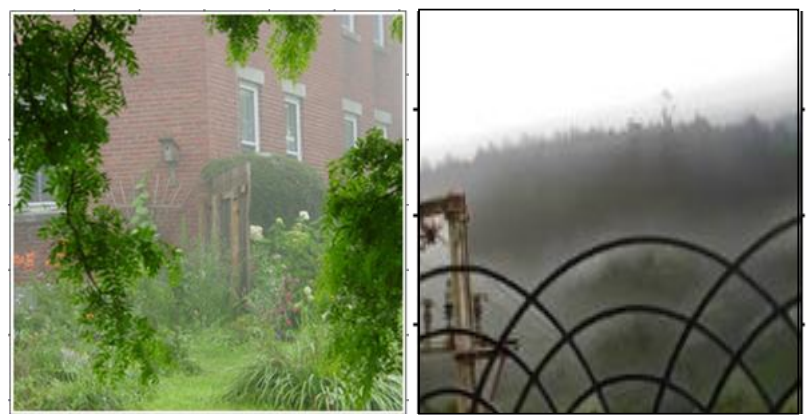

Fig 2: Original Image of House and Park

Fig 3: (a) Defoged Image by using Multi scale Fusion (b) Defoged Image by using Universal Defoging (c) Defoged Image after Guided filter

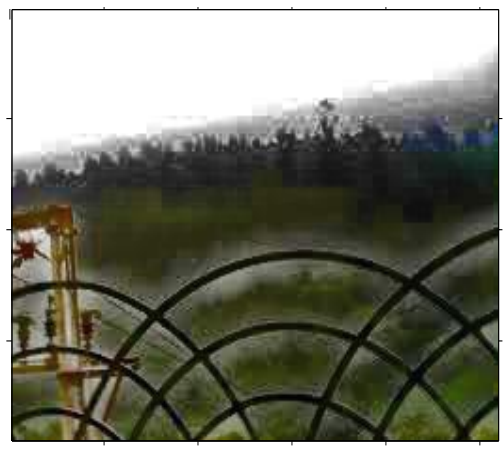

(a)

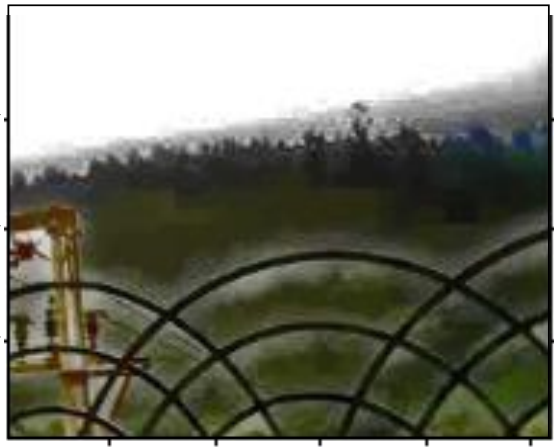

(b)

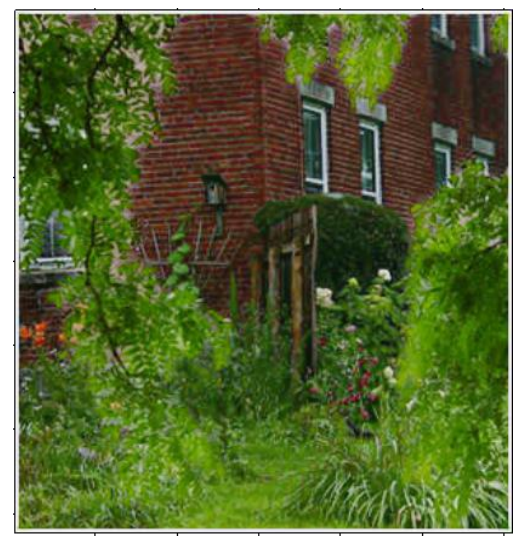

(c)

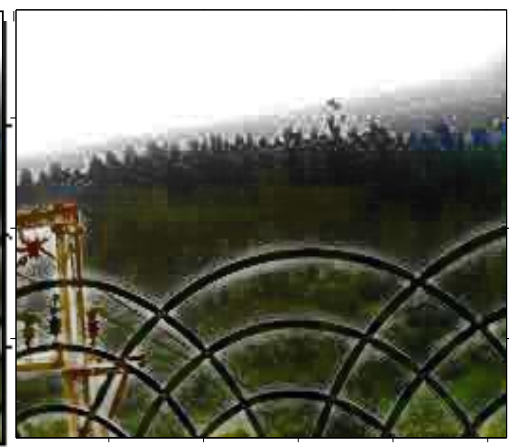

(c)

Fig 4: (a) Defoged Image by using Multi scale Fusion (b) Defoged Image by using Universal Defoging (c) Defoged Image after Guided filter.

Table 1. Comparison parameter for the house image

\begin{tabular}{|c|c|c|c|c|}
\hline METHOD & VARIANCE & MEAN & SNR & UIQI \\
\hline $\begin{array}{c}\text { Multiscale } \\
\text { fusion }\end{array}$ & 0.1039 & 0.5315 & 6.2288 & 2.7719 \\
\hline $\begin{array}{c}\text { Universal } \\
\text { dehazing with } \\
\text { guided filter }\end{array}$ & 0.1344 & 0.4342 & 6.0875 & 2.5927 \\
\hline
\end{tabular}

Table 2. Ccomparison parameter for the park image

\begin{tabular}{|c|c|c|c|c|}
\hline METHOD & VARIANCE & MEAN & SNR & UIQI \\
\hline $\begin{array}{c}\text { Multiscale } \\
\text { fusion }\end{array}$ & 0.0337 & 0.3913 & 6.2345 & 4.8957 \\
\hline $\begin{array}{c}\text { Universal } \\
\text { dehazing with } \\
\text { guided filter }\end{array}$ & 0.0407 & 0.3463 & 8.0638 & 6.5275 \\
\hline
\end{tabular}


It is implemented using MATLAB 7.9.0 (R2009b) on i-5 processor with 4-GB RAM. The simulations have been tested on aerial images in figure 3 and 4 . Figure 2 shows the Original Image of House image of haze removed Image. The parameter comparison is shown in the table 1 and 2 for the multiscale and universal defogging method.

\section{CONCLUSION AND FUTURE SCOPE}

In this paper, a fast and effective method for real-time image and video defoging is proposed. Using a newly presented image prior - dark channel prior, haze removal for a single image without using any extra information is formulated as a particular filtering problem and an improved filtering scheme is proposed based on guided filter. In the presented algorithm, the air-light and the down-sampled transmission can be estimated and extracted easily. Then using a guided filter, the transmission can be further refined and up-sampled. Results demonstrate the presented method abilities to remove the fog layer and achieve real-time performance. It is believed that many applications, such as outdoor surveillance systems, intelligent vehicle systems, remote sensing systems, graphics editors, etc. could benefit from the proposed method.

\section{REFERENCES}

[1] I. Yoon, S. Kim, D. Kim, M. H. Hayes, and J. Paik, "Adaptive defogging with color correction in the HSV color space for consumer surveillance system," IEEE Trans. on Consumer Electronics, vol. 58, no. 1, Feb. 2012, pp. 111-116.

[2] C. Zhen, S. Jihong, and P. Roth, "Single image defogging algorithm based on dark channel priority," Journal of Multimedia, vol. 8, no. 4, Aug. 2013, pp. 432438 .

[3] W. Feng, N. Guan, X. Zhang, X. Huang, and Z. Luo, "Single image defogging with single and multiple hybrid scattering model," International Conf. on Security, Pattern Analysis, and Cybernetics (SPAC), 18-19 Oct. 2014, pp. 247-252.

[4] H. Kawarabuki and K. Onoguchi, "Snowfall detection in a foggy scene," 22nd International Conf. on Pattern Recognition (ICPR), 24-28 Aug. 2014, pp. 877 - 882.

[5] Y. -K. Wang and C.-T. Fan, "Single image defogging by multiscale depth fusion," IEEE Trans. on Image Process., vol. 23, no. 11, Nov. 2014, pp. 4826-4837.

[6] Y. Lee, K. B. Gibson, Z. Lee, and T. Q. Nguyen, "Stereo image defogging," IEEE International Conf. on Image Processing (ICIP), 27-30 Oct. 2014, pp. 5427 - 5431.

[7] C. C. Cheng, F.-C. Cheng, P.-H. Lin, S.-C. Huang, "A L0 norm transmission model for defogging images," IEEE International Conf. on Consumer Electronics Taiwan, 2014, pp. 151 - 152.

[8] H. Zhao, C. Xiao, J. Yu, and X. Xu, "Single Image Fog Removal Based on Local Extrema," IEEE/CAA Journal of Automatica Sinica, vol. 2, no. 2, Apr. 2015, pp. 158165 .

[9] L. K. Choi, J. You, and A. C. Bovik, "Referenceless prediction of perceptual fog density and perceptual image defogging," IEEE Trans. on Image Process., vol. 24, no. 11, Nov. 2015, pp. 3888-3901.

[10] Y. Xu, J. Wen, L. Fei, and Z. Zhang, "Review of Video and Image Defogging Algorithms and Related Studies on
Image Restoration and Enhancement," IEEE Access, 2016, vol.: 4, pp. $165-188$.

[11] F. Fu and F. Liu, "Wavelet-based retinex algorithm for unmanned aerial vehicle image defogging," 8th International Symposium on Computational Intelligence and Design (ISCID), 2015, vol. 1, pp. 426 - 430.

[12] L. Deng, O.-X. Li, and S.-W. Zhao, "An improved image defogging algorithm based on global dark channel prior and fuzzy logic control," 12th International Computer Conf. on Wavelet Active Media Technology and Information Processing (ICCWAMTIP), 18-20 Dec. 2015, pp. 188 - 191.

[13] D. Sharma, Y. Kurmi, and V. Chaurasia, "Formation of super- resolution image: a review," Int. Jour. of Emerging Tech. and Adv. Engg., Apr. 2014, vol. 4, no. 4, pp. 218-221.

[14] Y. Kurmi and V. Chaurasia, "An image fusion approach based on adaptive fuzzy logic model with local level processing," Int. Jour. of Comp. Appl., Aug. 2015, vol. 124 , no.1, pp. 39-42.

[15] S. Tiwari, K. Chauhan, and Y. Kurmi "Shadow detection and compensation in aerial images using MATLAB," Int. Jour. of Comp. Appl., June 2015, vol. 119, no.20, pp. 59.

[16] Y. Kurmi and V. Chaurasia, "Performance of haze removal filter for hazy and noisy images," Int. Jour. of Sci. Engg. and Tech., Apr. 2014, vol. 3 no. 4, pp. 437 439.

[17] M. K. Patle, B. Chourasia, and Y. Kurmi, "High Dynamic Range Image Analysis through Various Tone Mapping Techniques," Int. Jour. of Comp. Appl., vol.153, no. 11, Nov. 2016 pp. 14-17.

[18] A. Kumar, B. Chourasia, and Y. Kurmi, "Image defogging by multiscale depth fusion and hybrid scattering model," International Journal of Computer Applications (0975 - 8887), vol. 155, no 11, Dec. 2016, pp. 34-38.

[19] C. Chengtao1, Z. Qiuyu, and L. Yanhua, "A Survey of Image Dehazing Approaches," 27th Chinese Control and Decision Conf. (CCDC), 2015, pp. 3964 - 3969.

[20] Fengzhi Fu; Fang Liu, "Wavelet-Based Retinex Algorithm for Unmanned Aerial Vehicle Image Defogging," 8th Inter. Symposium on Comput. Intell. and Design (ISCID), 2015, vol. 1, pp. 426-430.

[21] L. Deng; O. X. Li; S. W. Zhao, "An improved image defogging algorithm based on global dark channel prior and fuzzy logic control," 12th International Computer Conf. on Wavelet Active Media Tech. and Info. Process. (ICCWAMTIP), 2015, pp.188 - 191.

[22] L. Bo, X. Qingguo, "Inland river image defogging based on optimized contrast enhancement," IEEE Information Technology, Networking, Electronic and Automation Control Conf., 2016, pp. 145 - 150.

[23] H. Park, J. Park, H. Kim and J. Paik, "Improved DCPBased Image Defogging Using Stereo Images," 6th International Conf. on Consumer Electronics - Berlin (ICCE-Berlin), 2016, pp. 48 - 49. 
[24] Y. Lee, K. Hirakawa, and T. Q. Nguyen, "Joint defogging and demosaicking," IEEE Trans. on Image Process., 2016, vol. PP, no. 99, pp. 1 - 1.

[25] K. He, J. Sun, and X. Tang, "Single image haze removal using dark channel prior," in Proc. IEEE Conf. Comput. Vis. Pattern Recognit., Jun. 2009, pp. 1956-1963.

[26] L. Kratz and K. Nishino, "Factorizing scene albedo and depth from a single foggy image," in Proc. IEEE Int. Conf. Comput. Vis., Sep.-Oct. 2009, pp. 1701-1708.
[27] J.-P. Tarel and N. Hautiere, "Fast visibility restoration from a single color or gray level image," in Proc. IEEE Int. Conf. Comput. Vis., Sep.-Oct. 2009, pp. 2201-2208.

[28] C. O. Ancuti, C. Ancuti, and P. Bekaert, "Effective single image dehazing by fusion," in Proc. IEEE Int. Conf. Image Process., Sep. 2010, pp. 3541-3544.

[29] H. B. Mitchell, Image Fusion: Theories, Techniques and Applications. New York, NY, USA: Springer-Verlag, 2010. 